\title{
Extensive Training Promotes Performance Improvement but not Automaticity in Patients with Parkinson's disease
}

\author{
Piemonte MEP ${ }^{1^{*}}$, Oliveira TP ${ }^{1}$, Miranda $\mathrm{CS}^{1}$, Guelfi ETN ${ }^{1}$, Souza $\mathrm{CO}^{1}$, Okamoto $\mathrm{E}^{1}$, Xavier $\mathrm{GF}^{2}$ \\ ${ }^{1}$ Department of Physiotherapy, Communication Science \& Disorders, Occupational Therapy, School of Medicine, University of São Paulo, Brazil \\ ${ }^{2}$ Department of Physiology, Institute of Biosciences, University of São Paulo, Brazil
}

*Corresponding author: Maria Elisa Pimentel Piemonte. Department of Physiotherapy, Communication Science \& Disorders, Occupational Therapy, School of Medicine, University of São Paulo - USP, R. Cipotânea, 51, 05360-160, São Paulo, Brazil. Tel: + 5511 30917451; Fax: +55 11 30917461; E-mail: elisapp@usp.br

Received date: October 6, 2015; Accepted date: November 18, 2015; Published date: November 25, 2015

Copyright: $@ 2015$ Piemonte MEP et al. This is an open-access article distributed under the terms of the Creative Commons Attribution License, which permits unrestricted use, distribution, and reproduction in any medium, provided the original author and source are credited.

\begin{abstract}
Objective: This study evaluated the effects of extensive motor training in a finger-to-thumb opposition sequence task on performance and automaticity of patients at early and intermediate stages of Parkinson's disease (PD).

Methods: Fifteen PD patients in stage 1 of Hoehn and Yahr classification, 15 patients in stages 2 and 3, and 20 healthy matched control individuals, were extensively exposed to a 5-element finger-to-thumb opposition sequence task, 2 sessions per week, along 5 weeks. On session 1 the participants performed a specified sequence of movements along 60 seconds in a single-task condition; the number of sequences completed correctly was recorded. On sessions 2 to 9 the subjects were exposed to 600 repetitions per session of the same sequence of movements. On session 10 the participants performed 4 different 60 -second-duration trials including (1) the trained sequence and (2) a novel sequence, both in a single-task condition, and (3) the trained sequence and (4) a novel sequence, both concurrently with a verbal fluency task, therefore in a dual-task condition. The number of sequences completed correctly was recorded.
\end{abstract}

Results: All groups exhibited improvement of performance for the trained sequence. However, as expected, this improvement was relatively greater for the control subjects as compared to that seen for PD patients. Performance in the dual-task condition disrupted performance of all groups. However, while for control subjects this disruption was smaller for the trained as compared to the novel sequence, thus indicating automaticity for the trained sequence, for PD patients disruption was equivalent for trained and novel sequences.

Conclusion: Extensive motor training promotes improvement of performance in PD patients at different stages of the disease. However, this improvement is not associated with development of automaticity for the trained sequence. It seems important to take this information into account when planning therapeutic training approaches for PD patients.

Keywords: Parkinson's disease; Automatic control; Attention; Learning; Training, Basal ganglia

\section{Introduction}

Bradykinesia is among the early symptoms of Parkinson's disease (PD) and seems to be related to the patients' difficulties to perform learned motor skills automatically. In fact, loss of automatic control has been claimed to be one of the symptoms of PD [1]. Evidence from experimental and clinical studies has confirmed a progressive difficulty to perform learned movements automatically [2-4]. In addition to deficits in movement neural coding, there would be a failure to shift execution of movements to subcortical levels. Performance of motor skills along progression of PD has been associated with increments of activity in the cerebellum, premotor area, parietal cortex, precuneus and prefrontal cortex, since these figures differ from those seen in healthy subjects; those activations are interpreted as a strategy to compensate for the basal ganglia dysfunction [5]. Despite these neural compensatory mechanisms, the lack of automatic control of movement seem to result in impairments in gait, balance, facial expression, speech modulation, swallowing and routine tasks [6]. In other words, automatic control seems fundamental for efficient performance in these activities.

Deficits exhibited by PD patients during performance of dual-tasks have also been associated with lack of automatic control. That is, the patients tend to engage attentional control in order to compensate for the impaired automatic control of motor performance. Consequently, the remaining attentional resources are not enough to support performance of the secondary task. In other words, deficit of performance in dual-task condition may result from decline in automaticity [7].

Functionality for daily living activities dependent on the ability to perform learned movements under dual-task conditions. This would explain why PD patients exhibit difficulties for daily living activities, that is, their disturbance would be related to loss in automaticity.

Dan et al. showed that deficits in learning sequences of movements by $\mathrm{PD}$ patients is stronger in later stages of acquisition usually associated to development of automatic control. Since this effect occurs before the emergence of the early symptoms [8], it suggests that automaticity is among the early functions disturbed in PD. 
Page 2 of 6

Not surprisingly, attempts to minimize PD patients' functional difficulties in daily living activities not rarely involve training based on increased attentional control of movement. For instance, external cues, attentional and cognitive strategies have been used to improve performance in gait $[9,10]$, transfer [11,12], balance [13-15] and speech [16] in rehabilitation programs for these patients.

$\mathrm{Wu}$ and Hallet gathered evidence that even though slower as compared to elderly healthy subjects, PD patients achieve automaticity after training. Among 15 PD patients included in this study, 12 were capable of maintaining motor performance under a dual-task condition. However, this was only observed for simple sequences of movements, defined by the authors as a criterion for achievement of automatic control. In contrast, only 3 of these patients were considered to have achieved automatic control when complex sequences of movements were involved [2]. Wu et al. [7] discussed the potential of training strategies to promote recovery of automatic control on gait and balance, relying on the assumption that it is possible to recover automatic control through repetitive training.

Identification of the PD patients' ability to achieve automatic control is critical for orienting training approaches aiming at improving daily living activities and therefore quality of life of patients and their families.

This study investigated learning and automaticity resulting from extensive training in a finger-to-thumb opposition movement task involving sequences in PD patients at early and intermediate stages of the disease. The working hypothesis was that PD patients' improvement along repetitive training is related to attentional control and not to automaticity, independently on the stage of the disease.

\section{Methods}

\section{Participants}

Fifteen patients in early stage (stage 1) of PD (EPD Group) and 15 patients in intermediate stage (stages 2 and 3) of PD (IPD Group), according to the Hoehn and Yahr [17] classification, undergoing treatment with levodopa, were recruited from PD associations, following evaluation by an experienced specialist. Healthy volunteers $(\mathrm{N}=20)$ matched for age and gender, recruited from the same community, were assigned to the control group (CT). Inclusion criteria involved (1) idiopathic Parkinson's disease as diagnosed by an experienced specialist in movement disorders, following the UK Brain Bank criteria [18], (2) absence of neurological diseases (except for PD in EPD and IPD groups) and detectable sensory and/or motor disturbances in hands and arms, (3) a minimum score of 24 on the Mini-Mental State Examination [19], (4) normal or corrected visual acuity, and (5) lack of auditory losses. Patients and volunteers with extensive practice involving hand movements, such as musicians and typists, were excluded from the study. A total of 50 participants took part in the study including 28 men and 22 women (see details on Table 1 ), with mean age of $63.2+9.2$ years, right-hand dominance, and mean of $8+5$ years of formal education.

Patients were tested 40 to 120 minutes after their L-dopa dose (MED on condition), always at the same time of day.

This sample size design relied on a pilot study [20] showing that 12 patients per group is sufficient to a power greater than $0.9(\alpha=0.05$; $\mathrm{ES}=0.91)$.

\begin{tabular}{|l|l|l|l|}
\hline & $\begin{array}{l}\text { Control Group } \\
(\mathrm{n}=20)\end{array}$ & $\begin{array}{l}\text { Early PD patients } \\
\text { Group }(\mathrm{n}=15)\end{array}$ & $\begin{array}{l}\text { Intermediate PD } \\
\text { patients Group } \\
(\mathrm{n}=15)\end{array}$ \\
\hline Age (years) & $63.2 \pm 9.01$ & $63.7 \pm 9.27$ & $66.03 \pm 9.63$ \\
\hline Gender (male) & 12 & 8 & 8 \\
\hline $\begin{array}{l}\text { Disease duration } \\
\text { (years) }\end{array}$ & - & $3.61 \pm 1.98$ & $8.25 \pm 3.30$ \\
\hline $\begin{array}{l}\text { Motor exam (UPDRS } \\
- \text {-Section III) }\end{array}$ & - & $7.15 \pm 3.26$ & $22.25 \pm 7.20$ \\
\hline $\begin{array}{l}\text { Mini-Mental State } \\
\text { Examination }\end{array}$ & $28.2 \pm 1.2$ & $27.3 \pm 1.6$ & $26.7 \pm 1.9$ \\
\hline
\end{tabular}

Table 1: Age, as expressed by the mean number (+ standard deviation) of years, number of male subjects per group and scores achieved in the Mini-Mental Examination, by the Control participants and Early PD and Intermediate PD patients.

A written informed consent was signed for each participant. The present research protocol attends to national and international standards and policies, and was approved by the Ethics Committee of the Hospital das Clínicas da Universidade de São Paulo, São Paulo, Brazil (protocol 581/02).

\section{The Finger-to-thumb Opposition Sequence Task}

A finger-to-thumb opposition sequence task [21] was employed. The fingers of the hand were sequentially numbered 1 to 4 , being 1 the index finger and 4 the little finger. Two sequences of equal length and complexity were used; one sequence was the reverse of the other (4-1-3-2-4 and 4-2-3-1-4). One of these sequences was randomly assigned for the subjects of each group as either the trained sequence (TS) or new sequence (NS) in a counterbalanced schedule. The participants were instructed to tap each finger to the thumb following the informed sequence "as accurately and quickly as possible". Independent trials were run for each sequence and for each hand. The participants performed the task while seated comfortably in front of the desk where they could support their elbows and forearms. Speed and accuracy of performance were continuously monitored using a device attached to each fingertip and the thumb, connected to a computer programed to record each tap and the sequence of movements.

\section{Procedure}

Performance of each participant was assessed along 10 individual sessions. Since there were 2 sessions per week in distinct days, this assessment lasted for 5 weeks. The 1st and 10th sessions consisted of testing sessions before (BT) and after (AT) training, respectively (see below). Differently, the 2nd throughout the 9th sessions consisted of training sessions.

The participants were instructed to tap the informed sequence repetitively soon after presentation of the auditory stimulus and to stop doing it upon presentation of the same auditory stimulus. In case of errors of the tapping sequence, the subjects had to continue the task without pausing. 
Citation: Piemonte MEP, Oliveira TP, Miranda CS, Guelfi ETN, Souza CO et al. (2015) Extensive Training Promotes Performance Improvement but not Automaticity in Patients with Parkinson's disease. J Neurol Neurophysiol 6: 324. doi:10.4172/2155-9562.1000324

Page 3 of 6

\section{Before-training (BT) Testing}

The participants were exposed to the printed sequence of numbers assigned for them as TS, and asked to memorize it. After 2 minutes retention of the sequence was assessed by asking the participant to repeat it verbally. After accurate memorization, the participants performed the finger-to-thumb opposition sequence task until completing 10 repetitions of the sequence, in order to get familiarized with the task. Then, the BT testing was run along 60 seconds using the TS and the trained hand. They were asked to repeat the movements corresponding to the sequence "as accurately and quickly as possible". This task was performed without the concurrent performance of other tasks, which was named "single task (ST) condition". The number of sequences completed correctly was recorded as the dependent variable.

\section{Training}

Along training sessions (2nd throughout 9th sessions), the participants were re-exposed to the TS printed sequence and then asked to perform it following the same instruction provided during the BT testing, in a ST condition.

Each training session consisted of 600 repetitions of the TS, organized in 4 consecutive blocks of 120 repetitions each. There was a resting interval of 2 minutes after each block.

\section{After-training (AT) Testing}

Performance during after-training (AT) testing was evaluated in 4 distinct trials in which the TS and NS sequences were performed either alone (ST condition) or concurrently with another task [dual-task (DT) condition-see below]. Therefore, the AT testing involved evaluation of performance (1) using the TS in a ST condition, (2) using the TS in a dual-task (DT), (3) using the NS in a ST condition, and (4) using the NS in a DT condition, in this sequence. The intertrial interval was 2 minutes. The basic procedures employed in each case were the same as those employed in the BT testing, except for the inclusion of the DT condition.

The concurrent task employed for the DT condition involved a phonological form of verbal fluency task. That is, the participants had to state a load as many words as possible beginning with either " $\mathrm{F}$ ", "A" or "S" letters, concurrently with performance of the finger-to-thumb sequence task. Different letters were counterbalanced for participants within each group.

Comparisons of performance using the TS in a ST condition before and after the training allowed evaluation of the effect of repetitive training. Comparisons of performances in the ST conditions as compared to those seen in corresponding DT conditions allowed evaluation of automaticity.

\section{Data Analysis}

The Kolmogorov-Smirnov and Levene tests were used to assess normality and homogeneity of variance of the number of sequences performed correctly, respectively, for all measures.

The number of TS sequences correctly completed was subjected to a Repeated Measures Analysis of Variance (ANOVA), having Groups (CT, EPD and IPD) as the between subjects factor, and Testing Session (BT and AT) as the within subjects factor. This allowed evaluation of the effect of repetitive training on performance of TS.
As expected, performance of the finger-to-thumb sequence task under the DT condition was poorer as compared to the ST condition, as revealed by a reduction in the number of sequences performed correctly. This "cost effect" was expressed by taking the number of correct sequences performed in the ST condition as one hundred percent and by calculating the percentage of reduction of the corresponding score in the DT condition. Then, an additional ANOVA having Groups (CT, EPD and IPD) as the between subjects factor, and Sequence (TS and NS) as the within subjects factor was run for cost effects.

The effect sizes (ES) were calculated for all ANOVAs (alpha=0.05). A Tukey HSD post-hoc test was performed whenever required. Statistical software version 11.0 (StatSoft, USA) was used for all analyses. P-values smaller than 0.05 were considered statistically significant.

\section{Results}

\section{Effects of repetitive training}

Figure 1 shows the mean number of sequences performed correctly by the EPD, IPD and CT subjects, during performance of the trained sequence in a single-task condition, both in the BT and AT sessions.

The ANOVA including these scores revealed significant main effects for Groups (F2, 40=16.1, $\mathrm{P}<0.00001, \mathrm{ES}=0.99)$ and Testing Session (F1, $40=153.9, \mathrm{P}<0.000001, \mathrm{ES}=.99$ ), and a significant interaction effect for Groups $\mathrm{x}$ Training Session [F2, 40=8.28, $\mathrm{p}<0.001, \mathrm{ES}=0.94]$. This later effect seems to be related to a greater relative improvement by controls subjects as compared to both EPD and IPD groups. Post-hoc comparisons using the Tukey HSD test indicated that the mean number of correct sequences in the AT testing session was significantly greater when compared to the corresponding scores in the BT testing session, thus reflecting task acquisition by all groups. In addition, as expected, performance of the IPD group was significantly inferior as compared to CT scores, both in the BT and AT testing sessions. Furthermore, performance of EPD group was significantly inferior, as compared to CT subjects, in AT testing session but not in the BT testing session.

Thus, repetitive training lead to improvements of performance in a finger-to-thumb opposition sequence task, by both EPD and IPD patients, despite their inferior performance relative to that seen in healthy volunteers. In addition, the relative improvement of performance after training, as compared to the corresponding group's performance before training, was greater in the control subjects (Figure 1). 
Citation: Piemonte MEP, Oliveira TP, Miranda CS, Guelfi ETN, Souza CO et al. (2015) Extensive Training Promotes Performance Improvement but not Automaticity in Patients with Parkinson's disease. J Neurol Neurophysiol 6: 324. doi:10.4172/2155-9562.1000324

Page 4 of 6

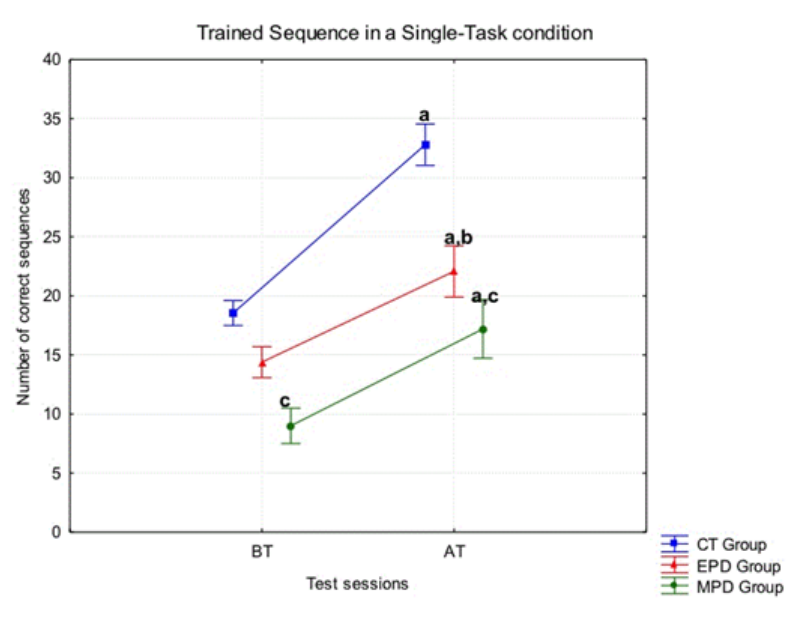

Figure 1: Mean number (+ S.E.M.) of the number of sequences performed correctly by the Early (EPD) and Intermediate $(\mathrm{MPD}=\mathrm{IPD}) \mathrm{PD}$ patients, and Control (CT) subjects, during performance of the trained sequence (TS) in a single-task (ST) condition, in testing sessions run before (BT) and after (AT) training. a. Post-hoc Tukey tests: before training $\mathrm{X}$ after training comparison: $\mathrm{p}<0.001$. b. Post-hoc Tukey tests: control group $\mathrm{x}$ early PD group comparison: $p<0.005$. c. Post-hoc Tukey tests: control group $\mathrm{x}$ intermediate $\mathrm{PD}$ group comparison: $\mathrm{p}<0.005$

\section{Disruption of performance with TS and NS sequences in the dual-task condition}

The present study evaluated automaticity effects by introducing a secondary concurrent verbal fluency task during performance of the principal finger-to-thumb sequence task, and compared performance in this dual-task condition with that seen when the principal task was performed alone.

The ANOVA including these cost effects revealed significant main effects for Groups $(\mathrm{F} 2,36=26.1, \mathrm{P}<0.000001, \mathrm{ES}=0.99)$ and Sequence $(\mathrm{F} 1,36=3.76, \mathrm{P}<0.05, \mathrm{ES}=0.61)$, and a significant interaction effect for Groups $\mathrm{x}$ Sequence [F2, 36=4.35, p< 0.01, ES=0.79]. Post-hoc comparisons using the Tukey HSD test indicated that the mean cost effects were inferior for TS in comparison to NS only for CT. In addition, surprisingly, the cost effects for TS and NS was similar for EPD and CT. Furthermore, cost effects of IPD group was significantly superior, as compared to CT and EPD subjects, for both sequences (TS and NS).

As can be seen in Figure 2 the cost effects exhibited by CT subjects in the dual task condition was stronger for the NS as compared to those seen for the TS, thus revealing automaticity rendered by repetitive training. In contrast, even though at different levels both EPD and IPD patients exhibited similar cost effects for both the TS and NS, thus indicating lack of automatization for the TS (Figure 2). Finally, note that IPD patients exhibited stronger cost effects for TS and NS as compared to the other groups (Figure 2) thus emphasizing that progression of the disease render PD patients more dependent on attentional resources.

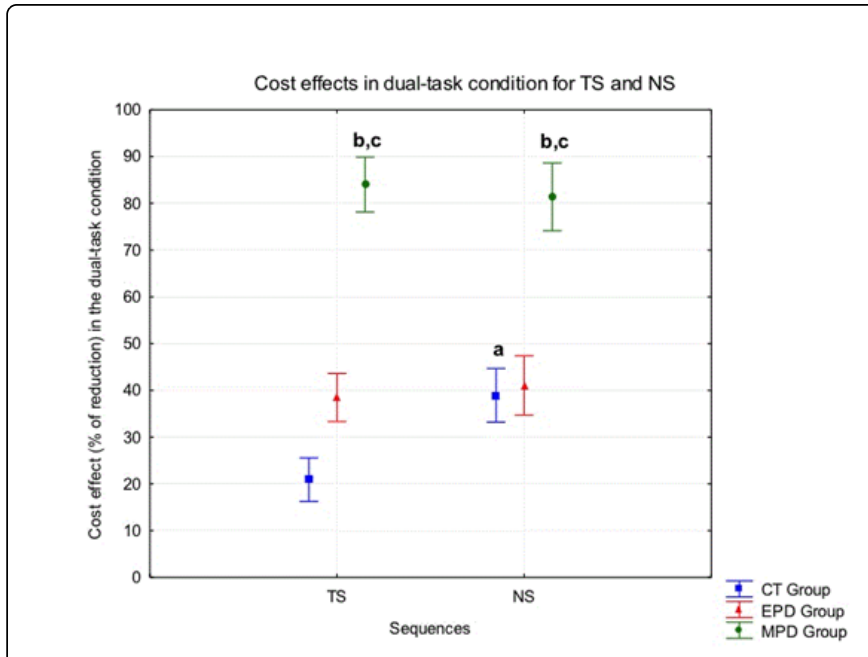

Figure 2: Mean percentage of reduction (+ S.E.M.) in the number of sequences performed correctly by the Early (EPD) and Intermediate (MPD=IPD) PD patients, and Control (CT) subjects, during performance of trained (TS) and novel (NS) sequences, in the dualtask condition relative to that seen in the single-task condition (performance in the corresponding single-task condition was taken as $100 \%)$. a. Post-hoc Tukey tests: TS X NS comparison: $\mathrm{p}<0.005$. b. Post-hoc Tukey tests: early PD group $\mathrm{x}$ intermediate PD group comparison: $\mathrm{p}<0.001$. c. Post-hoc Tukey tests: control group $\mathrm{x}$ intermediate PD group comparison: $\mathrm{p}<0.001$.

\section{Discussion}

This study investigated the ability of patients in early and intermediate stages of Parkinson's disease to learn a finger-to-thumb opposition sequence task by repetitive training, and to which extent these patients were capable of achieving automaticity.

Repetitive training led to improvement of performance for both EPD and IPD patients, and CT subjects (Figure 1). Data also showed that the proportion of improvement by EPD and IPD patients after training, relative to their performance before training, were similar (Figure 1). That is, even though exhibiting different basal levels of performance, the amount of improvement rendered by repetitive training were equivalent for both EPD and IPD groups. In contrast, the relative amount of improvement achieved by CT subjects was greater (Figure 1), confirming data or a prior study [22], rendering plausible to hypothesize that these subjects were getting the benefit of processes not available for PD patients. We propose that this process corresponds to automaticity provided by repetitive training.

This hypothesis was evaluated by comparing performance of EPD, IPD and CT subjects in the finger-to-thumb opposition sequence task using both an extensively trained sequence and a novel sequence, in a dual-task condition involving concurrent performance in of a verbal fluency task. If extensive repetitive training had rendered some degree of automaticity for the trained sequence the dual-task-induced cost effect on performance would be smaller as compared to that seen for the novel sequence. 
Results clearly showed that performance of both trained and novel sequences under the dual-task condition disturbs performance of $\mathrm{CT}$, EPD and IDP subjects, and that this disturbance was stronger for IDP subjects. In addition, there was a differential sequence effect in CT subjects, with minimal disturbance when testing in the dual-task condition involved the TS and a stronger disturbance when it involved the NS. This result suggests that automaticity provided by repetitive training partially prevents the disruptive effect induced by the dualtask condition. Interestingly, this differential sequence effect was not observed for both the EPD and IPD patients, thus indicating that even though these patients improve performance along repetitive training they do not get the benefit of automaticity.

In other words, extensive training for EPD and IPD patients was not enough to allow reduction of attentional control in order to perform both repetitive and novel sequences. These results have important implications for designing rehabilitation approaches for patients with Parkinson's disease.

Despite their motor difficulty, patients with Parkinson's disease are capable of improving motor performance following repetitive training, even when classified in intermediate stages of disease's progression. In other words, even though exhibiting an important reduction in movement speed as compared to patients in early stages of Parkinson's disease, patients in intermediate stages of the disease exhibited similar proportions of improvement following repetitive training.

Patients in early stage of the disease, despite reaching similar levels of performance relative to those seen in healthy participants before training (Figure 1), were unable to reach the same level of performance after the training. This might suggest an initial reduction in the learning process efficiency, as predicted by Pavão's et al. model [23], and suggest that patients with Parkinson's disease may require additional training in order to reach the same level of learning than healthy individuals [2].

In other words, extensive training can help patients in early and intermediate stages of Parkinson's disease progression to improve motor performance. Therefore, motor repetitive training for deficient movements might be considered a useful and effective strategy to minimize reduction of functional losses in these patients, thus helping to improve their quality of life.

There have been proposals that disruption of performance by PD patients in the dual-task condition is associated to executive dysfunction $[24,25]$. Data of the present study emphasized that PD patients engage attentional control in order to perform sequential movements independently on the degree of prior training with these movements. In addition, data involving EPD patients showed that their level of performance for the NS in a dual-task condition was similar to that seen for healthy volunteers, thus indicating that their ability to deal with the requirements of both tasks simultaneously was preserved. Therefore, disruption of PD patients in early stages of the disease in dual-task conditions seems more parsimoniously interpreted as reflecting a disruption of automaticity.

Thus, improvement of performance of these patients after training seems to be related to compensatory mechanisms dependent on attentional control, being the slight deficiency observed relative to healthy subjects possibly related to limitations in this kind of compensatory strategy.

In fact, movement automatization in healthy individuals seems related to increments in neural efficiency $[3,4,26,27]$. That is, automaticity has been associated with increments in effective connectivity between motor areas, including cerebellum, cingulate motor area, supplementary motor area, and posterior putamen. Patients with Parkinson's disease exhibit greater activations in the premotor area, parietal cortex, and cerebellum while performing repetitive movements, suggesting that compensatory mechanisms engage additional neural networks as compared to those of healthy people [28].

Neural correlates of repetitive movement in healthy subjects indicate the occurrence of a shift of control as the new motor skill become automatic, engaging the striatum. Patients at early stages of $\mathrm{PD}$ seem to adopt compensatory strategies to perform repetitive movements, because their automatization mode is not efficient [3].

Results of the present study involving dual-task condition confirm that patients in early stages of Parkinson's disease adopt compensatory strategies to perform repetitive and novel tasks. However, these strategies were not enough to sustain automatic control of the learned sequences. In other words, these patients have lost automaticity and this loss seems not possible to be reverted even after extensive repetitive training.

This conclusion, even though consistent with the data, requires confirmation, since a relatively small sample of patients participated in the present study. Additional studies involving greater samples of patients at different stages of the disease would be desirable.

The results of the present study bring important implications for the choice of therapeutic training approaches. Since loss of automaticity related to the disease cannot be reverted by extensive training, therapeutic approaches might focus on increasing compensatory mechanism, instead of insisting in recovery of automatic control.

\section{Conclusion}

Patients in early and intermediate stages of Parkinson's disease are capable of improving performance of sequential movements through repetitive training. However, they are not able to achieve automatic control of these movements.

\section{References}

1. Marsden CD (1980) Managing extrapyramidal disorders. Br Med J 281: 871.

2. Wu T, Hallett M (2005) A functional MRI study of automatic movements in patients with Parkinson's disease. Brain 128: 2250-2259.

3. Wu T, Liu J, Zhang H, Hallett M, Zheng Z1, et al. (2015) Attention to Automatic Movements in Parkinson's Disease: Modified Automatic Mode in the Striatum. Cereb Cortex 25: 3330-3342.

4. Lehéricy S, Benali H, Van de Moortele PF, Pélégrini-Issac M, Waechter T, et al. (2005) Distinct basal ganglia territories are engaged in early and advanced motor sequence learning. Proc Natl Acad Sci U S A 102: 12566-12571.

5. Wu T, Chan P, Hallett M (2010) Effective connectivity of neural networks in automatic movements in Parkinson's disease. Neuroimage 49: 2581-2587.

6. Peterson DS, Fling BW, Mancini M, Cohen RG, Nutt JG, et al. (2015) Dual-task interference and brain structural connectivity in people with Parkinson's disease who freeze. J Neurol Neurosurg Psychiatry 86: 786-792.

7. Wu T, Hallett M, Chan P (2015) Motor automaticity in Parkinson's disease. Neurobiol Dis 82: 226-234. 
Citation: Piemonte MEP, Oliveira TP, Miranda CS, Guelfi ETN, Souza CO et al. (2015) Extensive Training Promotes Performance Improvement but not Automaticity in Patients with Parkinson's disease. J Neurol Neurophysiol 6: 324. doi:10.4172/2155-9562.1000324

Page 6 of 6

8. Dan X, King BR, Doyon J, Chan P (2015) Motor Sequence Learning and Consolidation in Unilateral De Novo Patients with Parkinson's Disease. PLoS One 10: e0134291.

9. Vitório R, Lirani-Silva E, Pieruccini-Faria F, Moraes R, Gobbi LT, et al. (2014) Visual cues and gait improvement in Parkinson's disease: which piece of information is really important? Neuroscience 277: 273-280.

10. Brodie MA, Dean RT, Beijer TR, Canning CG, Smith ST, et al. (2015) Symmetry matched auditory cues improve gait steadiness in most people with Parkinson's disease but not in healthy older people. J Parkinsons Dis 5: 105-116.

11. Kamsma YPT, Brouwer WH, Lakke JPWF (1995) Training of compensatory strategies for impaired gross motor skills in patients with Parkinson's disease. Physiother Th Pract 11: 209-229.

12. Nieuwboer A, De Weerdt W, Dom R, Truyen M, Janssens L, et al. (2001) The effect of a home physiotherapy program for persons with Parkinson's disease. J Rehabil Med 33: 266-272.

13. Bhatt T, Yang F, Mak MK, Hui-Chan CW, Pai YC (2013) Effect of externally cued training on dynamic stability control during the sit-tostand task in people with Parkinson disease. Phys Ther 93: 492-503.

14. Capato TT, Tornai J, Ávila P, Barbosa ER, et al. (2015) Randomized controlled trial protocol: balance training with rhythmical cues to improve and maintain balance control in Parkinson's disease. BMC Neurol 15: 162.

15. Pompeu JE, Mendes FA, Silva KG, Lobo AM, Oliveira T de P, et al. (2012) Effect of Nintendo Wii ${ }^{\mathrm{Tn}}$-based motor and cognitive training on activities of daily living in patients with Parkinson's disease: a randomised clinical trial. Physiotherapy. 98: 196-204.

16. Kotz SA, Gunter TC (2015) Can rhythmic auditory cuing remediate language-related deficits in Parkinson's disease? Ann N Y Acad Sci 1337: 62-68.

17. Goetz CG, Poewe W, Rascol O, Sampaio C, Stebbins GT, et al. (2004) Movement Disorder Society Task Force report on the Hoehn and Yahr staging scale: status and recommendations. Mov Disord 19: 1020-1028.

18. Hughes AJ, Ben-Shlomo Y, Daniel SE, Lees AJ (1992) What features improve the accuracy of clinical diagnosis in Parkinson's disease: a clinicopathologic study. Neurology 42: 1142-1146.
19. Brucki SM, Nitrini R, Caramelli P, Bertolucci PH, Okamoto IH (2003) Suggestions for utilization of the mini-mental state examination in Brazil. Arq Neuropsiquiatr 61: 777-781.

20. Piemonte MEP, Souza CO, Neves ET, Okamoto E, Silva K, et al. (2004) Correlation between the severity of bradykinesia and the ability to learn a new motor skill in patients with Parkinson s disease. In: 8th International Congress of Parkinson's Disease and Movement Disorders, Roma. Movement Disorders. Hoboken: Wiley-Liss 19:39.

21. Karni A, Meyer G, Rey-Hipolito C, Jezzard P, Adams MM, et al. (1998) The Acquisition of Skilled Motor Performance: Fast and Slow ExperienceDriven Changes in Primary Motor Cortex. Proceedings of the National Academy of Science of the United States of America 95: 861-868.

22. dos Santos Mendes FA, Pompeu JE, Modenesi Lobo A, Guedes da Silva K, Oliveira T de P, et al. (2012) Motor learning, retention and transfer after virtual-reality-based training in Parkinson's disease - effect of motor and cognitive demands of games: a longitudinal, controlled clinical study. Physiother 98: 217-23.

23. Pavão R, Helene AF, Xavier GF (2012) Parkinson's disease progression: implicit acquisition, cognitive and motor impairments, and medication effects. Front Integr Neurosci 10: 6:56.

24. Dirnberger G, Jahanshahi M (2013) Executive dysfunction in Parkinson's disease: a review. J Neuropsychol 7: 193-224.

25. Rochester L, Galna B, Lord S, Burn D (2014) The nature of dual-task interference during gait in incident Parkinson's disease. Neuroscience 265: 83-94.

26. Poldrack RA, Sabb FW, Foerde K, Tom SM, Asarnow RF, et al. (2005) The neural correlates of motor skill automaticity. J Neurosci 25: 5356-5364.

27. Balsters JH, Ramnani N (2011) Cerebellar plasticity and the automation of first-order rules. J Neurosci 31: 2305-2312.

28. Adam JJ, Scholtissen B, Marí-Beffa P (2012) Response preparation in Parkinson's disease: automatic vs. controlled processing. Acta Psychol (Amst) 139: 465-470. 